\title{
Shrimp draft genome contains independent clusters of ancient and current endogenous viral elements (EVE) of the parvovirus IHHNV
}

\author{
Suparat Taengchaiyaphum ${ }^{1}$, Prapatsorn Wongkhaluang ${ }^{1}$, Timothy W. Flegel ${ }^{2,3}$ and \\ Kallaya Sritunyalucksana ${ }^{1 *}$
}

\begin{abstract}
${ }^{1}$ Aquatic Animal Health Research Team (AQHT), Integrative Aquaculture Biotechnology Research Group, National Center for Genetic Engineering and Biotechnology (BIOTEC), National Science and Technology Development Agency (NSTDA), Yothi office, Rama VI Rd., Ratchathewi, Bangkok 10400, Thailand

${ }^{2}$ Center for Excellence in Shrimp Molecular Biology and Biotechnology (Centex Shrimp), Faculty of Science, Mahidol University, Rama VI Rd., Ratchathewi, Bangkok 10400, Thailand ${ }^{3}$ National Center for Genetic Engineering and Biotechnology (BIOTEC), National Science and Technology Development Agency (NSTDA), 113 Paholyothin Rd., Khlong Luang, Pathum Thani, 12120 Thailand
\end{abstract}

\section{*Corresponding author:}

Dr. Kallaya Sritunyalucksana (Email: kallaya@ biotec.or.th)

\section{Abbreviations:}

IHHNV: Infectious hypodermal and haematopoietic necrosis virus, IHHNV-A: non-infectious IHHNV Type A, cvcDNA: circular viral copy DNA, RT-PCR: reverse-transcriptase polymerase chain reaction, EVE: endogenous viral element(s), piRNA: PIWI-interacting RNA(s), RNAi: RNA interference

\section{ABSTRACT}

\section{Background}

Shrimp have the ability to accommodate viruses in long term, persistent infections without signs of disease. Endogenous viral elements (EVE) play a role in this process probably via production 
of negative-sense Piwi-interacting RNA (piRNA)-like fragments. These bind with Piwi proteins to dampen viral replication via the RNA interference (RNAi) pathway. We searched a draft genome of the giant tiger shrimp (Penaeus monodon)(GenBank record JABERT000000000) for the presence of EVE related to a shrimp parvovirus originally named infectious hypodermal and hematopoietic necrosis virus (IHHNV).

\section{Results}

The shrimp draft genome contained 3 piRNA-like gene clusters containing scrambled IHHNV EVE. Two clusters were located distant from one another in linkage group 35 (LG35). Both LG35 clusters contained multiple DNA fragments with high homology (99\%) to GenBank records DQ228358 and EU675312 that were both called "non-infectious IHHNV Type A" (IHHNV-A) when originally discovered. However, our results and those from a recent Australian P. monodon genome assembly indicate that the relevant GenBank records for IHHNV-A are sequenceassembly artifacts derived from scrambled and fragmental IHHNV-EVE. Although the EVE in the two LG35 clusters showed high homology only to IHHNV-A, the clusters were separate and distinct with respect to the arrangement (i.e., order and reading direction) and proportional content of the IHHNV-A GenBank records. We conjecture that these 2 clusters may constitute independent allele-like clusters on a pair of homologous chromosomes. The third EVE cluster was found in linkage group 7 (LG7). It contained EVE with high homology (99\%) only to GenBank record AF218266 with the potential to protect shrimp against infectious IHHNV.

\section{Conclusions}

54 Our results suggested the possibility of viral-type specificity in EVE clusters. Specificity is 55 important because whole EVE clusters for one viral type would be transmitted to offspring as collective hereditary units. This would be advantageous if one or more of the EVE within the cluster were protective against disease caused by the cognate virus. It would also facilitate gene editing for removal of non-protective EVE clusters or for transfer of protective EVE clusters to genetically improve existing shrimp breeding stocks that might lack them.

Key words: Viral accommodation, Endogenous viral element(s) (EVE), Infectious hypodermal 


\section{BACKGROUND}

64 Non-retroviral viral gene sequences inserted into host genomes during the course of evolution began to be called endogenous viral elements (EVE) upon their discovery in vertebrate genomes [1]. However, evidence for EVE in animals was first reported for insects in 1999 [2], but the significance of the paper was not fully appreciated at the time of its publication. For shrimp, an EVE was described much later [3] but it was named "non-infectious IHHNV".

In 2009, it was hypothesized that EVE (at the time called non-retroviral viral genome inserts) resulted from a mechanism that provided for heritable immunity in shrimp and insects [4, 5]. In brief, the mechanism was proposed to involve host recognition of invading viral mRNA followed by its use as a substrate for host reverse transcriptase (RT) to produce variable fragments of viral copy DNA (vcDNA). The vcDNA fragments would subsequently be inserted into the shrimp genome where they would produce antisense RNA that could induce an RNA interference (RNAi) response. This would lead to inhibition of viral replication and allow the host to accommodate one or more viruses (i.e., allow viral accommodation) in persistent infections without signs of disease. Tests for predictions of the viral accommodation hypothesis proceeded much faster with insects than shrimp. By 2020, the basic predictions regarding EVE were proven for mosquitoes [6-8]. The studies in both Drosophila and mosquitoes revealed additional detailed mechanisms for an unpredicted, specific adaptive response involving the ability of vcDNA to produce siRNA resulting in an immediate, adaptive cellular and systemic antiviral RNAi response $[7,9,10]$. For details see a recent review [5].

One of the insect publications [10] revealed that the vcDNA produced by host RT in response to RNA virus infection came in two forms, one linear (lvcDNA) and one circular (cvcDNA). The latter could be specifically isolated relatively easily and was shown, when injected, to protect insect hosts againt the homologous virus. Following the protocols described for cvcDNA isolation in insects, it was shown [11] that IHHNV-cvcDNA could be extracted from IHHNV-infected $P$. monodon and that it could inhibit IHHNV replication in whiteleg shrimp $P$. vannamei challenged with IHHNV prepared from the infected P. monodon. During sequencing of the cvcDNA extract,

92 it was also found that some of the cvcDNA constructs had high homology (98-99\%) to the 
(i.e., from EVE) originally called "non-infectious IHHNV Type-A" (IHHNV-A) [3, 12]. Thus, the 2021 report [11] revealed that EVE can also give rise to cvcDNA, and it was hypothesized that the crcDNA arose via EVE-produced RNA interacting with host reverse transcriptase.

Around the time that the work with IHHNV cvcDNA [11] was being done, a draft whole genome sequence (WGS) of a Thai P. monodon specimen was published [13], and we became interested to determine whether the WGS might contain EVE related to IHHNV. We considered this possible because the specimes we used for our cvcDNA work and the specimen used for the WGS project originated from the same domesticated shrimp breeding stock.

103

\section{RESULTS AND DISCUSSION}

\section{Clusters of IHHNV-EVE were found in linkage groups 35 and 7}

A general BlastN search of the Thai P. monodon WGS project (GenBank accession no. JABERT000000000) [13] using the queries GenBank record DQ228358 for non- infectious IHHNV-A and GenBank record AF218266 for infectious IHHNV confirmed the presence of 3 clusters of EVE derived from IHHNV (Fig. 1). Two of these IHHNV clusters (Fig. 1A) were located in linkage group 35 (LG35) and showed high sequence homology (98-99\%) to IHHNV in GenBank accession numbers DQ228358 and EU675312. The other cluster (Fig. 1B) was located in linkage group 7 (LG7) and showed high sequence homology (98-99\%) to GenBank accession number AF218266 for an extant type of infectious IHHNV. All three EVE clusters were clearly demarked by bracketing, direct host repeat sequences marked by red arrows in Fig. 1.

\section{Characteristics of EVE homologous to non-infectious IHHNV-A in LG35}

117 The GenBank records for DQ228358 (in P. monodon from East Africa) and EU675312 (in $P$. monodon from Australia) were initially referred to as "non-infectious IHHNV Type A [3, 12] even though the authors knew that the sequences originated from the host shrimp genomes ( $P$. monodon). The IHHNV-related portions of the records for DQ228358 and EU675312 share 99\% sequence identity, indicating that they arose from a similar, ancient type(s) of IHHNV that is

122 distantly related to the types of IHHNV currently reported to cause disease in cultivated shrimp

123 [14]. We now refer to the two GenBank records DQ228358 and EU675312 as EVE of non- 
125 primarily on the portion of the sequence ( 1 to $3025 \mathrm{bp}$ ) that has homology to IHHNV. The

126 remainder of the record (3026-4655 bp) constitutes shrimp host repeat and transposable element

127 sequences.

128

129

130

(A) LG35 EVE Cluster 1 homologous to non-infectious IHHNV-A
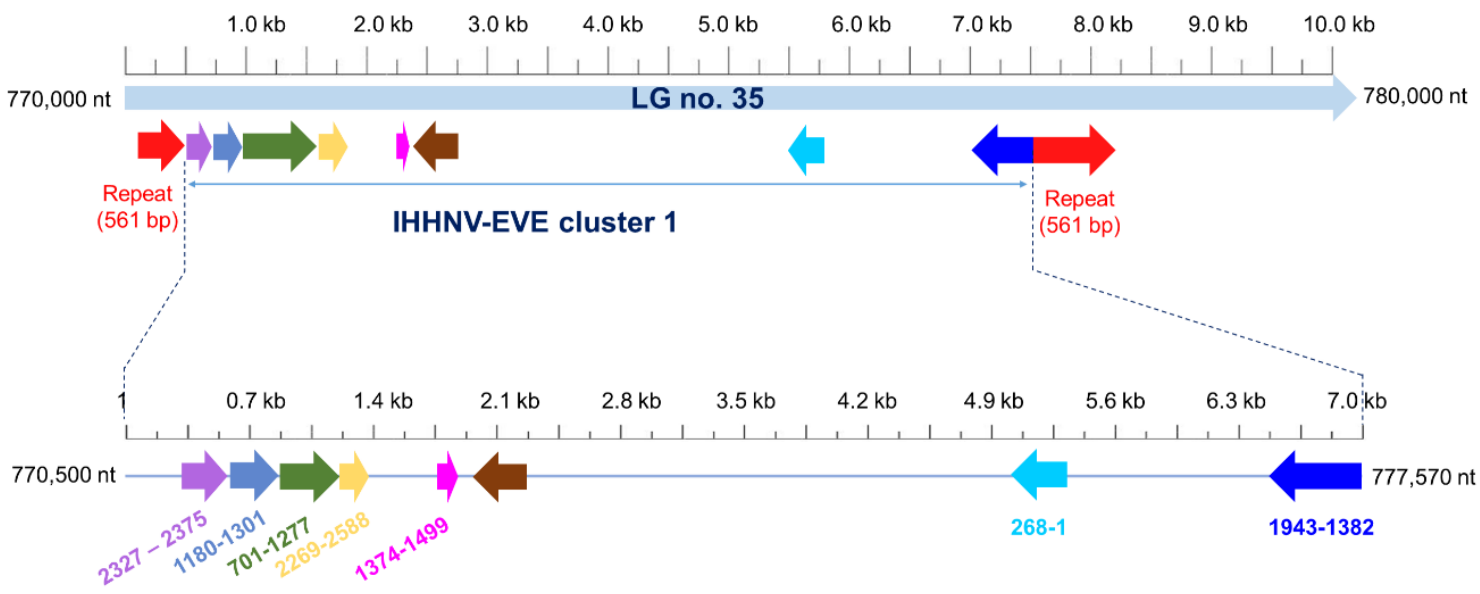

(B) LG35 EVE Cluster 2 homologous to non-infectious IHHNV-A

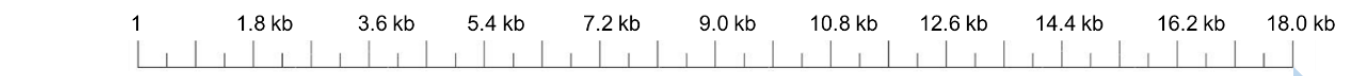

$862,000 \mathrm{nt}$

LG no. 35
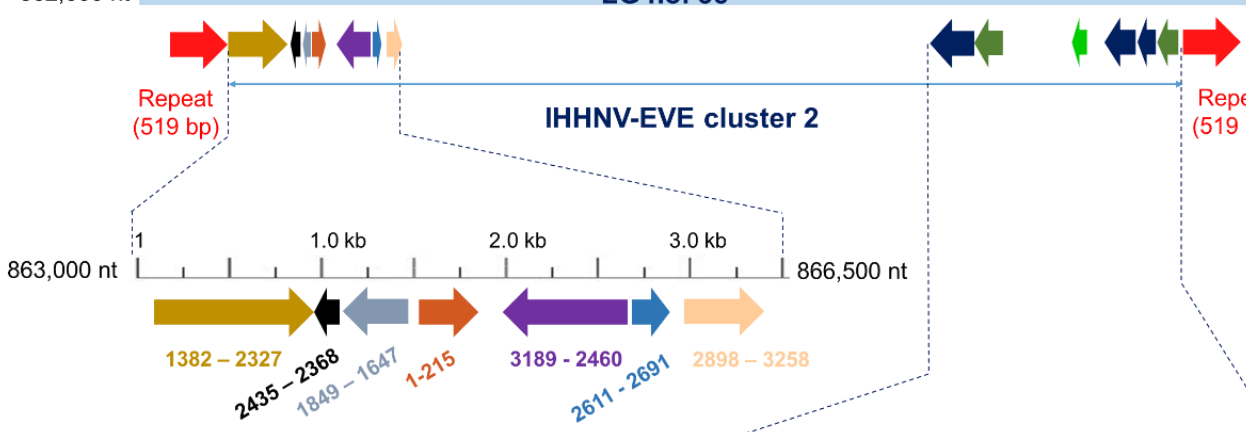
(C) LG7 EVE Cluster homologous to infectious IHHNV

157

158

159

160

161

162

163

164

165

166

167

168

169

170

171

172

173

174

175

176

177

178

179

180

181

182

183

184

185

186

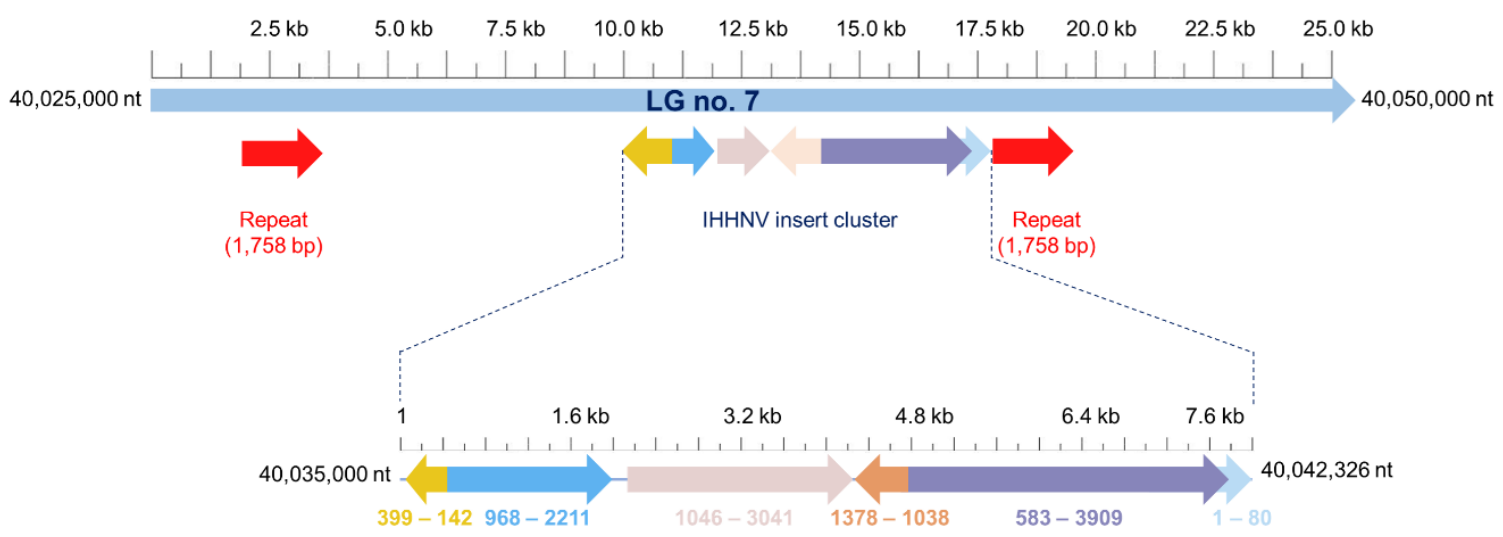

Figure 1. Schematic diagrams of IHHNV-EVE clusters in the draft Penaeus monodon WGS.

(A) and (B) Sequence diagrams for Cluster 1 and 2, respectively, in LG35 with high homology to the non-infectious IHHNV-A query portion (1-3025 bp) of the GenBank record DQ228358. Some of the EVE sequences in the 2 clusters correspond to the same region of DQ228358 (i.e., the same color) but may differ in length and in reading direction indicated by arrowheads. Others are unique to each cluster. A zoom- in expansion of each EVE cluster is shown beneath. Colored numbers below indicate the nucleotide positions corresponding to GenBank record DQ228358. The portion of the record related to the host transposable-element portion of DQ228358 is indicated by a dark brown arrow. (C) Diagram of the IHHNV-EVE cluster in LG7 (GenBank accession no. JABERT010000007.1) with EVE showing high sequence identity (99\%) to GenBank record AF218266 for infectious IHHNV. The numbers below the arrows represent the matching positions AF218266.

We were not surprised to find EVE of IHHNV-A in the Thai $P$. monodon WGS database. This was because we had already reported the occurrence of a variety of cvcDNA sequences with high sequence identity (98-99\%) to non-infectious IHHNV-A in P. monodon specimens obtained from a different generation of the same breeding stock from which the Thai genome specimen was obtained [11]. Those cvcDNA sequences covered 68\% (779-3930 nt) of the matching portion of the DQ228358 record (including some transposable element portion) and could be assembled into a single linear construct. However, at the time of that publication, we did not know the actual composition of the genome region(s) from which the cvcDNA sequences arose. All we could 
187

188

189

190

191

192

193

194

195

196

197

198

199

200

201

202

203

204

205

206

207

208

209

210

211

212

213

214

215

216

conclude at the time was that a variety of IHHNV-cvcDNA types were produced, some linked with host transposable element sequences and some not.

Our search of the Thai WGS revealed that LG35 did not contain a single continuous sequence with high sequence identity to IHHNV-A. Instead, it contained many high-identity EVE fragments of variable length that were scrambled with respect to proportion, position and reading direction in the non-infectious IHHNV-A reference sequences. These scrambled EVE were arranged in LG35 in two distinct clusters separated from one another by more than 80,000 bp (Figs. 1A and 1B).

The individual EVE are shown in different colors to allow easy visual comparison of the EVE to matching regions of the IHHNV portion of the DQ228358 reference sequence. The positions of the EVE in LG35 are shown below each cluster diagram in black typeface while the positions relative to the DQ228358 record are shown above in colors to match those of each EVE. The colored vertical lines indicate the boundaries of the individual EVE. The number and lengths of the individual EVE and the gaps between them in the 2 clusters in LG35 are shown in Table 1. The EVE in the two LG35 clusters contain some common DQ228358 coverage, but they are distinct from one another in terms of the EVE types, lengths and reading directions when compared to the DQ228358 reference sequence (Figs. 1A and 1B). The lengths of the EVE clusters bracketed by their respective repeat sequences were 6,744 bp for Cluster 1 is and 14,993 bp for Cluster 2.

The unexpanded diagrams for Clusters 1 and 2 in Fig. 1A and 1B shared some structural features. For example, both of the 2 EVE clusters (Fig. 1A and 1B) were bracketed by a pair of host, directrepeat sequences (red arrows) of 2 x 561 bp in Cluster 1 Fig. 1A) and 2 x 519 bp in Cluster 2 (Fig. 1B). In Cluster 1, the two repeats shared 98\% sequence identity (547/561 bp) (Supplementary Fig. S2) while those in Cluster 2 shared only 77\% sequence identity (400/519 bp)(Supplementary Fig. S3). These bracketing direct repeat sequences (red arrows) demarked the boundaries of the noninfectious IHHNV-A EVE clusters. They matched many other regions in several linkage groups of the Thai WGS database but at relatively low sequence identities (around $80 \%$ or less). 
217 Table 1. Lengths of the EVE and intervening sequences in the 3 clusters found in the draft WGS 218 of Thai P. monodon.

\begin{tabular}{|c|c|c|c|c|c|}
\hline \multicolumn{3}{|c|}{ LG35 Cluster 1 related to DQ228358 } & \multicolumn{3}{|c|}{ LG35 Cluster 2 related to DQ228358 } \\
\hline EVE no. & Size (bp) & Gap (bp) & EVE no. & Size (bp) & Gap (bp) \\
\hline 1 & 48 & 8 & 1 & 945 & 2 \\
\hline 2 & 118 & 3 & 2 & 67 & 8 \\
\hline 3 & 576 & 0 & 3 & 202 & 234 \\
\hline 4 & 319 & 348 & 4 & 729 & 189 \\
\hline 5 & 125 & 128 & 5 & 215 & 14 \\
\hline 6 & 204 & 2923 & 6 & 80 & 194 \\
\hline 7 & 267 & & 7 & 360 & 8125 \\
\hline Mean & 237 & 568 & 8 & 498 & 0 \\
\hline \multirow[t]{2}{*}{ SD } & 176 & 1161 & 9 & 319 & 1169 \\
\hline & & & 10 & 125 & 521 \\
\hline \multicolumn{3}{|c|}{ LG7 Cluster 3 related to AF218266 } & 11 & 128 & 43 \\
\hline EVE no. & Size (bp) & Gap (bp) & 12 & 206 & 15 \\
\hline 1 & 257 & 0 & 13 & 576 & \\
\hline 2 & 1243 & 101 & Mean & 342 & 876 \\
\hline 3 & 1995 & 2 & SD & 272 & 2308 \\
\hline 4 & 340 & 0 & & & \\
\hline 5 & 3326 & 0 & & & \\
\hline 6 & 80 & & & & \\
\hline Mean & 1207 & 21 & & & \\
\hline SD & 1268 & 45 & & & \\
\hline
\end{tabular}

Within the boundaries of Cluster 1 there was a host retrotransposon element (dark brown arrow) of $561 \mathrm{bp}$ (Fig. 1A) that had 98\% sequence identity to a matching portion of the $1395 \mathrm{bp}$ host retrotransposon sequence that is part of GenBank record DQ228358. However, there was also a hit for 1354/1395 bases ( $98 \%$ identity) for the same retrotransposon portion of the DQ228358 sequence beginning very distantly from Cluster 1 at position 23,321,662 in LG35. All other spaces that separated the EVE fragments in Cluster 1 showed homology to various other shrimp host retrotransposon elements or host repeat sequences (not shown).

229 In contrast to Cluster 1, the pattern for Cluster 2 in LG35 (Fig. 2B) did not include any retrotransposon sequence with high homology to that in GenBank record DQ228358 within the 
231 bracketing 519 bp direct repeat sequences (red arrows). Like Cluster 1, all sequences separating

232

233

234

235

236

237

238

239

240

241

242

243

244

245

246

247

248

249

250

251

252

253

254

255

256

257

258

259

260

261

the EVE showed homology to a variety of other shrimp host retrotransposon and repeat sequences (not shown). The bracketing sequences for EVE Clusters 1 and 2, matched many other regions in several linkage groups of the Thai WGS database but at lower sequence identities of around $80 \%$ or less (not shown).

It is not known whether these two clusters in LG35 are linked on one chromosome of a diploid pair or whether they are matching "allele-like clusters" ("cluster-alleles") located one each in the chromosome pair represented by LG35. Now that we have the sequences of the two clusters in LG35, it will be possible to design specific primers to screen for each type in the current generation of the original $P$. monodon stock. By identifying one specimen positive for both clusters and mating it with one negative for both, obtaining about half the offspring each carrying only one or the other cluster would confirm whether or not they are cluster-alleles. Finally, the forms of these 2 clusters resemble those described from mosquitoes as piRNA-like gene clusters containing fragmented sequences from mosquito RNA viruses [6, 7].

A preprint [15] using DNA extracted from an Australian P. monodon specimen for genome analysis revealed one EVE cluster in their Scaffold group 97 (SG97) with high identity to GenBank EU675312 (i.e., the Australian version of IHHNV-A with 99\% sequence identity to DQ228358). Given the information from our earlier publication and from the work reported herein and from Australia, it is likely that the continuous sequence records for DQ228358 and EU675312 at GenBank are assembly artifacts, and that they were obtained from fragmented and scrambled target sequences that had sufficient overlap to result in their assembly into single linear sequences when using the sequence of infectious IHHNV (GenBank record AF218266) as a reference [3, 12].

The preprint of Huerlimann et al. [15] gives detailed analyses of the EVE cluster found on SG97 of their Australian P. monodon specimen plus detailed comparison of it to the two EVE clusters that they (like us) discovered in LG35 of the Thai $P$. monodon WGS. Thus, those interested the detailed comparison should consult the Australian preprint. 


\section{The IHHNV-EVE in LG7 showed high sequence homology to infectious IHHNV}

263 Of greatest interest to us was the EVE cluster located in LG7 (Fig. 1 C) that showed high sequence identity (95-99\%) only to GenBank accession number AF218266 from an extant form of infectious IHHNV [17]. In other words, this cluster contained no EVE with high sequence identity to GenBank records DQ228358 and EU675312 (i.e., "non-infectious IHHNV Type A) that were found in EVE Clusters 1 and 2 in LG35. However, the general, overall pattern for EVE Cluster 3 was similar to those of the two for IHHNV-A EVE clusters located on LG35. For example, the LG7 EVE were scrambled in terms of fragment location, portion and reading direction with respect to the AF218266 reference genome. They were also bracketed by two host direct-repeat sequences

271 (red arrows) of 1,758 bp (98\% sequence identity). The EVE were either contiguous or separated

272 by host retrotransposon or repeat sequences. Again, the overall arrangement resembled the PiRNA-like gene clusters reported for EVE of RNA viruses in mosquitoes [6, 7].

Our work with IHHNV-cvcDNA [11] and the work with mosquitoes [7,8], suggest that negative sense RNA transcripts of the IHHNV-EVE in this cluster would have the potential capability of inducing a host RNAi response against infection by homologous types of infectious IHHNV. If our conjecture above that the 2 IHHNV-EVE in LG35 are alleles turns out to be correct, the fact that there is only one IHHNV-EVE cluster in LG7 suggests that it might be a single cluster/allele with no matching IHHNV-EVE on the homologous chromosome. If this is so, it would provide an opportunity to prove whether or not the IHHNV-EVE in LG7 is protective against infectious IHHNV.

For example, primers could be designed to screen for the IHHNV-EVE cluster in LG7 in the current generation of the $P$. monodon stock. Several cluster-positive individuals could be mated with several cluster- negative individuals. If the cluster behaves in an allele- like manner, subsequent screening of the offspring from these crosses would reveal that at least one of the positive parents carried a single copy of the LG7 cluster/allele and would yield half the offspring positive for the allele and half negative for it. The offspring could then be challenged with infectious IHHNV followed by qPCR to determine infectious IHHNV loads in shrimp with and

291 without the EVE cluster. A significantly lower mean IHHNV load in the cluster-positive offspring 
293

294

295

296

297

298

299

300

301

302

303

304

305

306

307

308

309

310

311

312

313

314

315

316

317

318

319

320

321

322

323

stock owner would then be able to use PCR to select crosses to maintain the protective cluster in subsequent stock generations.

\section{Detailed analysis of the IHHNV-EVE in LG7}

Altogether, the IHHNV-EVE cluster in LG7 (Fig. 1B) is 7,267 bp in length and contains 6 EVE having high sequence identity with a current type of infectious IHHNV (GenBank record AF218266. The EVE range from 80 to 3326 bp in length (Table 1) and are scrambled with respect to portion, position and reading direction when compared to the IHHNV reference genome AF218266. In comparison to the two EVE clusters for non- infectious IHHNV in LG35, 3 of the EVE in Cluster 3 are much longer and the gaps between the EVE are shorter or do not exist. The significance of these differences is currently unknown. However, the general pattern of EVE scrambling, bracketing by host repeat sequences and separation or not by host transposable element or repeat sequences is similar that in the other 2 EVE clusters in LG35.

\section{Trade consequences from shrimp stocks carrying an LG7 protective IHHNV-EVE}

We carried out a Blastn analysis of the IHHNV-EVE cluster in LG7 against the target sequences for the PCR detection method (“309”) recommended for specific detection of infectious IHHNV in the OIE diagnostic manual [18]. The 309 method was designed to be specific for detection of infectious IHHNV [12]. Our analysis of the EVE cluster in LG7 revealed that 3 of the EVE (2, 3 and 5) contained sequences with 95\% identity and 100\% coverage for the target of the 309 method based on the AF218266 reference sequence (Table 2). However, despite the less than 100\% overall sequence identities of the potential EVE targets, the primer sequences for the 309 method matched $100 \%$ with the relevant target sequences in all 3 of the potential EVE targets (Supplementary information 1). Thus, the potential amplicons would be equal length (indistinguishable) from those that would arise from infectious IHHNV and for the specimen used for the genome project would constitute a false positive test results for infectious IHHNV. 
Table 2. Potential targets in the IHHNV-EVE cluster of LG7. See the supplementary information for detailed information on the BlastN results.

\begin{tabular}{|c|c|c|c|c|}
\hline $\begin{array}{l}\text { Detection } \\
\text { Primer }\end{array}$ & $\begin{array}{l}\text { Primer } \\
\text { sequences }\end{array}$ & Hit positions on LG 7 & $\begin{array}{l}\text { Target } \\
\text { size } \\
\text { /identity }\end{array}$ & $\begin{array}{c}\text { Primer } \\
\text { Mismatch }\end{array}$ \\
\hline \multirow{3}{*}{ OIE [18] } & \multirow{3}{*}{ IHHNV309F/R } & Location $1: 40,036,120-40,036,428 \mathrm{nt}$ & $309 \mathrm{bp} / 95 \%$ & $0 / 22$ \\
\hline & & Location 2: 40,037,386 - 40,037,694 nt & $309 \mathrm{bp} / 95 \%$ & $0 / 22$ \\
\hline & & Location 3: 40,040,184-40,040,492 nt & $309 \mathrm{bp} / 95 \%$ & $0 / 22$ \\
\hline \multirow{2}{*}{$\begin{array}{l}\text { Jaroenram and } \\
\text { Owens, } 2014 \text { [19] }\end{array}$} & RPA1F & Location 1: 40,041,559-40,041,588 nt & $121 / 97 \%$ & $1 / 30$ \\
\hline & RPA1R & Location 1: 40,041,679-40,041,650 nt & $121 / 97 \%$ & $1 / 30$ \\
\hline \multirow{9}{*}{$\begin{array}{l}\text { Cowley et al., } \\
2018 \text { [20] }\end{array}$} & \multirow{3}{*}{$\begin{array}{l}\text { IHHNV- } \\
\text { q309F1 }\end{array}$} & Location 1: 40,036,089 - 40,036,115 nt & $98 / 100 \%$ & $0 / 27$ \\
\hline & & Location $2: 40,037,355-40,037,381 \mathrm{nt}$ & $98 / 100 \%$ & $0 / 27$ \\
\hline & & Location 3: 40,040,153-40,040,179 nt & $98 / 100 \%$ & $0 / 27$ \\
\hline & \multirow{3}{*}{$\begin{array}{l}\text { IHHNV- } \\
\text { q309R1 }\end{array}$} & Location 1: 40,036,186-40,036,161 nt & $98 / 100 \%$ & $0 / 26$ \\
\hline & & Location $2: 40,037,452-40,037,427 \mathrm{nt}$ & $98 / 100 \%$ & $0 / 26$ \\
\hline & & Location $3: 40,040,250-40,040,225 \mathrm{nt}$ & $98 / 100 \%$ & $0 / 26$ \\
\hline & IHHNV- & Location 1: 40,036,119-40,036,136 nt & NA & $0 / 18$ \\
\hline & q309Pr1 & Location $2: 40,037,385-40,037,402 \mathrm{nt}$ & NA & $0 / 18$ \\
\hline & (probe) & Location 3: $40,040,183-40,040,200 \mathrm{nt}$ & NA & $0 / 18$ \\
\hline
\end{tabular}

In addition to the recommended method in the OIE manual, there are two published methods proposed to aid in the detection of infectious IHHNV while avoiding false positives arising from IHHNV-EVE. One of these [19] is an isothermal PCR detection method focused on a part of the infectious IHHNV genome that appeared to be of low prevalence or absent from IHHNV-EVE revealed by screening $P$. monodon from Australia. Our analysis of the target sequence for the primers from this method in the LG7 EVE for infections IHHNV (Table 2) revealed only one target sequence in the cluster in which there was a single base mismatch for each primer. There was a low calculated effect on the binding efficiency of the primers, so it is hard to predict whether or not the method would give a false positive test for infectious IHHNV with the potential LG7 target. In contrast, a more recent qPCR method designed to avoid false positive test results for infectious IHHNV that arise from IHHNV-EVE [20] would not be effective with the LG7 EVE cluster because there are 3 target sequences with 100\% match to the primer and probe sequences. the methods above would have been considered IHHNV-infected and the positive shrimp 
specimens would have been discarded during the screening process to develop specific pathogen free (SPF) breeding stocks. In this way, it is possible that potentially protective EVE were discarded during stock development. This problem of false positive test results for infectious IHHNV arising from EVE has been raised previously [14, 21].

In addition, it is likely that shrimp products carrying the LG7 cluster would give false positive test results for the presence of infectious IHHNV, and this might result in the rejection of exported shrimp broodstock, shrimp PL and frozen shrimp products by countries that use the target of the OIE-recommended PCR method to screen imports for the presence of infectious IHHNV. It might be argued that use of a PCR method to detect such EVE followed by breeding selection to eliminate them from breeding stocks would be the simplest way to avoid this problem. However, such an approach might also remove naturally evolved, heritable resistance to IHHNV infection and lead to production problems for shrimp farmers.

In addition to the above potential for false positive results, another possibility has previously been raised [21]. This might occur in an SPF stock that had been developed by use of an internationally standardized PCR method to confirm absence of a particular virus based on a mutually agreed, small fragment of the target virus genome. This process would result not only in the discard of infected shrimp but also shrimp that carry EVE containing the PCR target sequence. Since some EVE may be protective and others not, it would be wise to choose the target region of any standard method to be in a genome sequence known to have no or low potential as a protective EVE.

However, this may not solve all the problems because individual shrimp in the stock population would carry a variety of difference EVE cluster/alleles for a particular virus. Thus, it is possible that 2 individuals in a stock population might each carry one of $2 \mathrm{EVE}$ in different cluster/alleles but in the same reading direction and have the potential to re-establish the PCR detection target by crossover. Specifically, the separated EVE fragments in the two shrimp would have to have sequence overlap but with the EVE in one individual containing the forward 5' primer target sequence for the chosen standard method but lacking the 3 ' primer sequence, while the other individual would contain the 3' primer sequence but lack the 5' sequence. These EVE would escape the screening process to remove stock individuals with EVE that carry the target for the 
standard PCR detection method chosen. However, at some unpredictable time in many crosses and generations, these to sequences might end up as cluter/alleles in a single individual by random assortment of chromosomes from its parents. If so, crossover events might occur in the overlap region to re-establish the PCR target sequence and give rise to a small portion of PCR positive individuals (i.e., "pop-up" positive individuals) in the offspring of such individuals. This could happen even in a population of shrimp with a good history of freedom from the target virus [21]. For these reasons, it is essential that development of standard PCR detection methods involve a process of investigation and consultation among regulatory agencies and the companies or agencies that develop and maintain SPF shrimp breeding stocks for shrimp farmers.

\section{The evolutionary advantage of jumbled contents of EVE clusters}

We believe that the characteristic scrambling of EVE in PiRNA-like clusters when compared to their arrangements in the originating genome in both insects and shrimp is worthy of some contemplation. We propose that this phenomenon may have evolved because it prevents the easy re-establishment of complete, infectious viral genome sequences in the host genome by the process of recombination between EVE-cluster alleles. On the other hand, the ability of the EVE to produce RNA transcripts and give rise to vcDNA [11] would seem to open the possibility that rare recombination events might occur between infecting viruses and EVE products (RNA or DNA) and be an additional potential source viral variation. As far as we know, this possibility has not been explored in shrimp or insects. Is it possible, for example, that the origin of viruses in the "hybrid" family Bidnaviridae [22-24] might have originated by a fortuitous recombination event between distinct EVE cluster/alleles that brought together EVE fragments from 2 different virus families into a novel, functional combination that led to the emergence of flacherie disease in the silkworm?

\section{CONCLUSIONS}

Revelation of piRNA- gene- like clusters of EVE from specific viral types is worth further investigation. This may exemplify an advantageous evolutionary development that arose because collections of EVE from specific viral types into single linkage clusters would assure their transmission to offspring as potential "protective antiviral EVE packages" (PEVEP). It would also an evolutionary advantage to have these PEVEP located on different chromosomes to assure that 
maximum variation in PEVEP combinations would occur simply by random assortment of chromosomes during the production of gametes. If such PEVEP operated like alleles, additional variation would be possible via crossover during meiosis. Without viral genome fragmentation and scrambling (including reading direction) during the formation of these piRNA gene-like clusters, it might be possible that crossover between two suitable APP would occasionally reestablish a full, infectious viral genome or even give rise to new virulent types. Fracture and jumbling may have evolved to circumvent this possibility. However, it does not eliminate the possibly of pop-up, false-positive PCR amplicons that might arise from crossover between EVE that re-establish the sequence of a PCR target for a standard viral detection method. Nor does it eliminate the possibility of RNA and vcDNA arising from EVE might on very rare occasions be incorporated into novel functional entities (e.g., Bidnaviridae) or contribute to the evolution of infectious viruses via recombination events.

\section{MATERIALS AND METHODS}

\section{Bioinformatics analysis of a draft $P$. monodon genome for IHHNV}

The sequences of non-infectious IHHNV (GenBank record DQ228358) and infectious IHHNV (GenBank record AF218266) were used as the subject reference sequences for BlastN analysis (https://blast.ncbi.nlm.nih.gov/Blast.cgi) of a recently released whole draft genome (WDG) of the giant tiger shrimp P. monodon (GenBank accession no. JABERT000000000) [13]. Prediction of conserved DNA repeat sequences was determined using the Dfam database of repetitive DNA families (https://www.dfam.org/home) [25].

\section{Conflict of interest}

Authors declare that there is no conflict of interest in this article.

\section{Acknowledgements}

This research project is supported by Mahidol University (Fundamental Fund: Basic Research Fund: fiscal year 2022) (Grant no. BRF1-054/2565) and National Center for Genetic Engineering and Biotechnology (BIOTEC), National Science and Technology Development Agency (NSTDA). 


\section{References}

1. Feschotte C, Gilbert CJNRG: Endogenous viruses: insights into viral evolution and impact on host biology. 2012, 13(4):283-296.

2. Lin CL, Lee JC, Chen SS, Wood HA, Li ML, Li CF, Chao YC: Persistent Hz-1 virus infection in insect cells: evidence for insertion of viral DNA into host chromosomes and viral infection in a latent status. J Virol 1999, 73(1):128-139.

3. Tang KF, Lightner DV: Infectious hypodermal and hematopoietic necrosis virus (IHHNV)-related sequences in the genome of the black tiger prawn Penaeus monodon from Africa and Australia. Virus Res 2006, 118(1-2):185-191.

4. Flegel TW: Hypothesis for heritable, anti-viral immunity in crustaceans and insects. Biology Direct 2009, 4(1):32.

5. Flegel TW: Research progress on viral accommodation 2009 to 2019. Dev Comp Immunol 2020, 112:103771.

6. Whitfield ZJ, Dolan PT, Kunitomi M, Tassetto M, Seetin MG, Oh S, Heiner C, Paxinos E, Andino R: The Diversity, Structure, and Function of Heritable Adaptive Immunity Sequences in the Aedes aegypti Genome. Curr Biol 2017, 27(22):3511-3519 e3517.

7. Tassetto M, Kunitomi M, Whitfield ZJ, Dolan PT, Sanchez-Vargas I, Garcia-Knight M, Ribiero I, Chen T, Olson KE, Andino R: Control of RNA viruses in mosquito cells through the acquisition of vDNA and endogenous viral elements. Elife 2019, 8.

8. Suzuki Y, Baidaliuk A, Miesen P, Frangeul L, Crist AB, Merkling SH, Fontaine A, Lequime S, Moltini-Conclois I, Blanc H et al: Non-retroviral Endogenous Viral Element Limits Cognate Virus Replication in Aedes aegypti Ovaries. Curr Biol 2020, 30(18):34953506 e3496.

9. Saleh MC, Tassetto M, van Rij RP, Goic B, Gausson V, Berry B, Jacquier C, Antoniewski C, Andino R: Antiviral immunity in Drosophila requires systemic RNA interference spread. Nature 2009, 458(7236):346-350.

10. Poirier EZ, Goic B, Tome-Poderti L, Frangeul L, Boussier J, Gausson V, Blanc H, Vallet T, Loyd H, Levi LI et al: Dicer-2-Dependent Generation of Viral DNA from Defective Genomes of RNA Viruses Modulates Antiviral Immunity in Insects. Cell Host Microbe 2018, 23(3):353-365 e358. 
11. Taengchaiyaphum S, Buathongkam P, Sukthaworn S, Wongkhaluang P, Sritunyalucksana K, Flegel TW: Shrimp Parvovirus Circular DNA Fragments Arise From Both Endogenous Viral Elements and the Infecting Virus. Front Immunol 2021, 12(3979).

12. Tang KF, Navarro SA, Lightner DV: PCR assay for discriminating between infectious hypodermal and hematopoietic necrosis virus (IHHNV) and virus-related sequences in the genome of Penaeus monodon. Dis Aquat Organ 2007, 74(2):165-170.

13. Uengwetwanit T, Pootakham W, Nookaew I, Sonthirod C, Angthong P, Sittikankaew K, Rungrassamee W, Arayamethakorn S, Wongsurawat T, Jenjaroenpun $\mathrm{P}$ et al: A chromosome- level assembly of the black tiger shrimp (Penaeus monodon) genome facilitates the identification of growth- associated genes. Mol Ecol Resour 2021, 21(5):1620-1640.

14. Saksmerprome V, Jitrakorn S, Chayaburakul K, Laiphrom S, Boonsua K, Flegel TW: Additional random, single to multiple genome fragments of Penaeus stylirostris densovirus in the giant tiger shrimp genome have implications for viral disease diagnosis. Virus Res 2011, 160(1-2):180-190.

15. Huerlimann R, Cowley JA, Wade NM, Wang Y, Kasinadhuni N, Chan C-KK, Jabbari J, Siemering K, Gordon L, Tinning M et al: Genome assembly of the Australian black tiger shrimp (Penaeus monodon) reveals a fragmented IHHNV EVE sequence. bioRxiv 2021:2021.2011.2011.468259.

17. Bonami JR, Trumper B, Mari J, Brehelin M, Lightner DV: Purification and characterization of the infectious hypodermal and haematopoietic necrosis virus of penaeid shrimps. J Gen Virol 1990, 71 ( Pt 11):2657-2664.

18. Infection with infectious hypodermal and haematopoietic necrosis virus. In: Manual of Diagnostic Tests for Aquatic Animals. 8 edn: OIE World Organisation for Animal Health; 2021: 138-153.

19. Jaroenram, W., Owens, L., 2014. Recombinase polymerase amplification combined with a lateral flow dipstick for discriminating between infectious Penaeus stylirostris densovirus and virus-related sequences in shrimp genome. J. Virol. Methods. 208, 144151. 
494 20. Cowley, J.A., Rao, M., Coman, G. J., 2018. Real-time PCR tests to specifically detect

495

496

497

498

499

500

501

502

503

504

505

506

507

508

509

510

511

512

513

514

515

516

517

518

519

520

521

522 IHHNV lineages and an IHHNV EVE integrated in the genome of Penaeus monodon. Dis. Aquat. Organ. 129, 145-158.

21. Alday-Sanz V, Brock J, Flegel TW, McIntosh R, Bondad-Reantaso MG, Salazar M, Subasinghe R: Facts, truths and myths about SPF shrimp in Aquaculture. Rev Aquacult 2020, 12(1):76-84.

22. Hu ZY, Li GH, Li GT, Yao Q, Chen KP: Bombyx mori bidensovirus: The type species of the new genus Bidensovirus in the new family Bidnaviridae. Chinese Sci Bull 2013, 58(36):4528-4532.

23. Krupovic M, Koonin EV: Evolution of eukaryotic single-stranded DNA viruses of the Bidnaviridae family from genes of four other groups of widely different viruses. Sci Rep $2014,4$.

24. Tijssen P, Penzes JJ, Yu Q, Pham HT, Bergoin M: Diversity of small, single-stranded DNA viruses of invertebrates and their chaotic evolutionary past. J Invert Pathol 2016, 140:8396.

25. Hubley R, Finn RD, Clements J, Eddy SR, Jones TA, Bao W, Smit AFA, Wheeler TJ: The Dfam database of repetitive DNA families. Nucleic Acids Res 2016, 44(D1):D81-D89.

3

14

515

16

.




\section{Supplementary information}

524

525

526

527

528

529

530

531

532

533

534

535

536

537

538

539

540

541

542

543

544

545

546

547

548

549
AF218266-309bp

R1:40036120-40036428 nt 1 R2: $40037386-40037694$ nt R3: 40040184-40040492 nt

AF218266-309bp

R1:40036120-40036428 nt R2: 40037386-40037694 nt R3: 40040184-40040492 nt

AF218266-309bp

R1: 40036120-40036428 nt R2: 40037386-40037694 nt R3: 40040184-40040492 nt

AF218266-309bp

R1:40036120-40036428 nt R2: 40037386-40037694 nt

R3: 40040184-40040492 nt

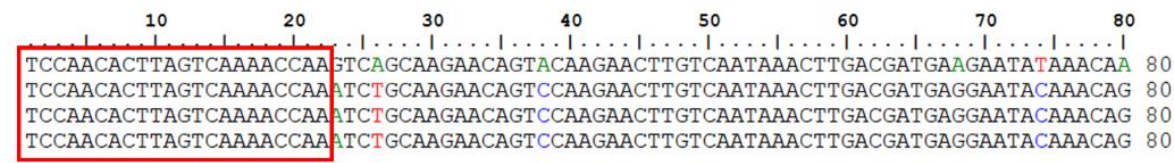

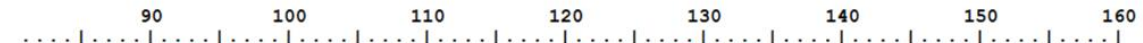

CTATGGACCCGTACCAGAGGA CAATATAAAGACAAACTCAGAGGAATATTAACATACTACAACAACAAGAAAAAGTCAAA 160 CTATGGACCCGCACCAGAGGACAATATAAAGACAAACTCAGGGGAATACTAACTTACTACAACAACAAGAAAAAGTCGAA 160 CTATGGACCCGCACCAGAGGACAATATAAAGACAAACTCAGGGGAATACTAACTTACTACAACAACAAGAAAAAGTCGAA 160 CTATGGACCCGCACCAGAGGACAATATAAAGACAAACTCAGGGGAATACTAACTTACTACAACAACAAGAAAAAGTCGAA 160

$\begin{array}{cccccccc}170 & 180 & 190 & 200 & 210 & 220 & 230 & 240\end{array}$ 161 C.AAAGCCAACTGTCACTAATTACAAACCTGCAGAATATATCCAAAAGAAACCAGACTACGACAATATGCAGTGGATAA 240 161 CCAAAGCCAACTGTCACTCATTACAAACCTGCAAAATATATCAAAAAGGAAACCAGACTACGACAACATGCAGTGGATAA 240 161 CCAAAGCCAACTGTCACTCATTACAAACCTGCAAAATATATCAAAAAGGAAACCAGACTACGACAACATGCAGTGGATAA 240 161 CCAAAGCCAACTGTCACTCATTACAAACCTGCAAAATATATCAAAAAGGAAACCAGACTACGACAACATGCAGTGGATAA 240

Supplementary figure S1. Multiple sequence alignment of potential $309 \mathrm{bp}$ targets for the OIErecommend IHHNV detection method from three repeats in IHHNV-EVE observed in LG 7 (R1$\mathrm{R} 3$ ). The $309 \mathrm{~F} / \mathrm{R}$ primer positions are indicated by red rectangles. The primer sequences and potential amplicon sizes match $100 \%$ to those expected for infectious IHHNV.

241 AATACATGTTAGCCAACAACGACATCCGTGTACCAGAATCTTAGCITGGATAATCATCGTAGCAGACA 309

241 AGTACATGTTAGCCAACAACGACATCCGTGTACCAGAAATCTTAGC TGGATAATCATCGTAGCAGACA 309

241 AGTACATGTTAGCCAACAACGACATCCGTGTACCAGAAATCTTAGC TGGATAATCATCGTAGCAGACA 309

241 AGTACATGTTAGCCAACAACGACATCCGTGTACCAGAAATCTTAGCT TGGATAATCATCGTAGCAGACA 309

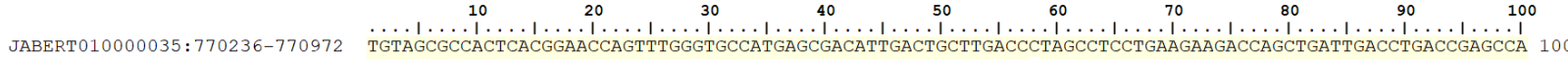
JABERT010000035:777568-778123 TGTAGCGCCACTCACGGAACCAGTTTGGGTGCCATGAGCGACATTGACTGCTTGACC-TAGCCTCCTGAAGAAGACCAGCTGATTGACCTGACCGAGCCA 99

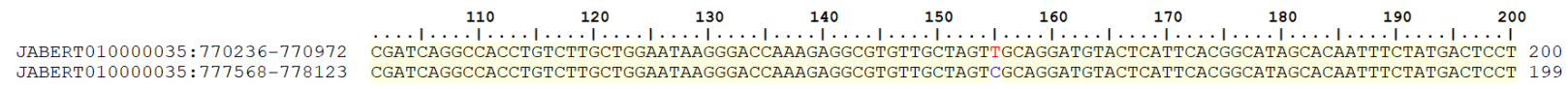

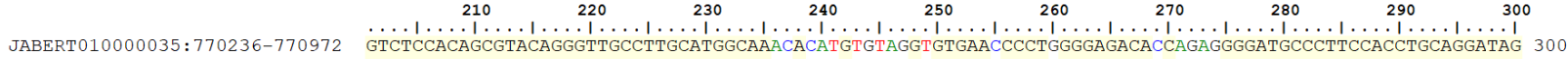
JABERT010000035:777568-778123 GTCTCCACAGCGTACAGGGTTGCCTTGCATGGCAACAATGGGCCATAGAGTGAACCCTCGGGAGACAGCCAGAGGGATGCCCTTCCACCTGCAGGATAG 299

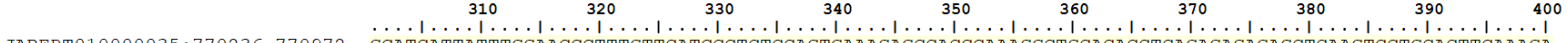
JABERT010000035: 770236-770972 GCATCATTATTTGGAACCCTTTGTTCATCCCTCTCCAGTGAAAGAGCCACCCAAAGGCTGCACACCTCA GAGAGA GAGCTCAAGTCCTGCACTTCAAAGA 400 JABERT010000035:777568-778123 GCATCATTATTTGGAACCCTTTGTTCATCCCTCTCCAGTGAAAGAGCCACCCAAAGGCTGCACACCTCAGAGAGAGAGCTCAAGTCCTGCACTTCAAAGA 399

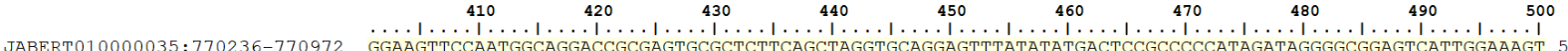
JABERT010000035:777568-778123 GGAATTCCAATGGCAGGACCGCGAGTGCGCTCTTCAGCTAGGTGCAGGAGTTTATATATGACTCCGCCCCCATAGATAGGGGCGGAGTCATTGGAAAGT 499

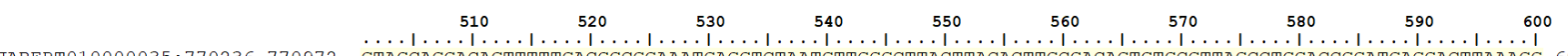
JABERT

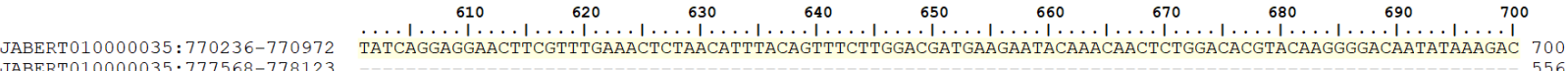
JABERT010000035:777568-778123

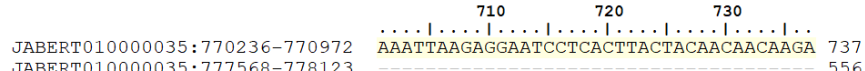


bioRxiv preprint doi: https://doi.org/10.1101/2021.12.29.474436; this version posted January 6, 2022. The copyright holder for this preprint (which was not certified by peer review) is the author/funder, who has granted bioRxiv a license to display the preprint in perpetuity. It is made available under aCC-BY-ND 4.0 International license.

550 Supplementary figure S2. Alignment of the $561 \mathrm{bp}$ repetitive sequences flanking the non-

551

552

553

554

555

556

557

558

559

560

561

562

563

564

565

566

567

568

569

570

571

572

573

574

575

576

577

578

579

8

9

55

76

579 infectious IHHNV-EVE Cluster 1 located in LG35. The sequences are aligned in the same direction and share $98 \%$ identity (547/561 bp with 9/561 nucleotide gaps).

JABERT010000035:862628-863097

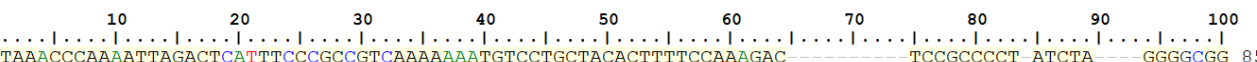
(⿸丆口 TAAGCCCAACATTAGACTTCATTTCGGCG - -TAAAACTCTGTCCTGCTACACTTT_CCAATGACGCACCGGTCGTCCGCCCCTTATCTAATGGGGGCTGG 97

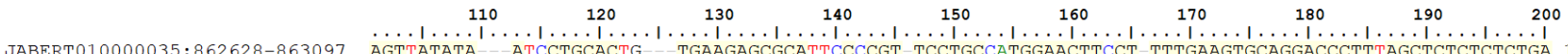

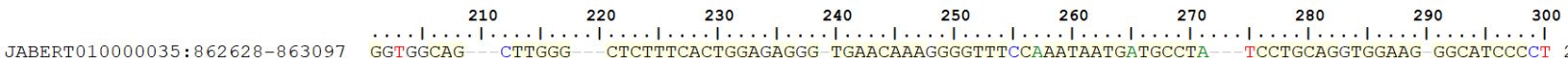

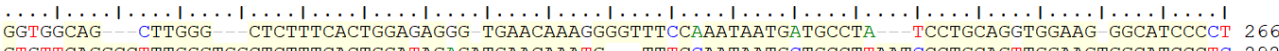

(20)

GGCH

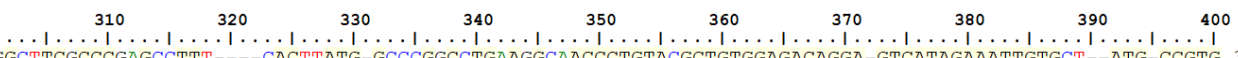

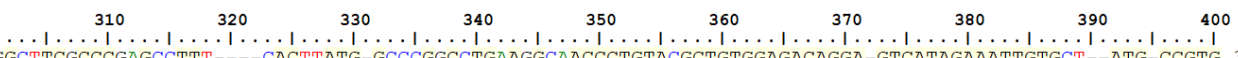

CTGGATAGAGAT

JABERT010000035: $862628-863097$

JABERT010000035:878369-878880

GTGTCTCCCAGGGGTTCACACTACACATGTGTTTGCCATGAGGAACCCACACTGTGGAGACAGGA-GTCATAGAAATTGTGCT-ATG-CCGTG 357

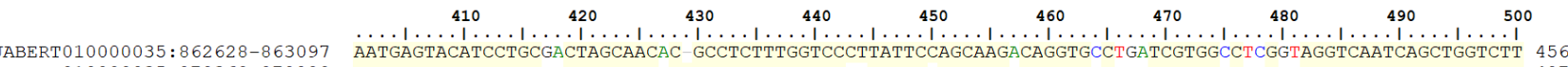

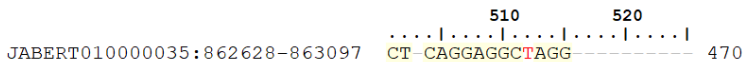

JABERT010000035:878369-878880 CTTCAGGAGGCGAGGGTCAAGCAGT 510

Supplementary figure S3. Alignment of the two $519 \mathrm{bp}$ repetitive sequences flanking IHHNVEVE Cluster 2 located on LG35. The sequences are aligned in the same direction and share $77 \%$ identity (400/519 bp with 66/519 nucleotide gaps).

50

71

74 
bioRxiv preprint doi: https://doi.org/10.1101/2021.12.29.474436; this version posted January 6, 2022. The copyright holder for this preprint (which was not certified by peer review) is the author/funder, who has granted bioRxiv a license to display the preprint in perpetuity. It is made available under aCC-BY-ND 4.0 International license.

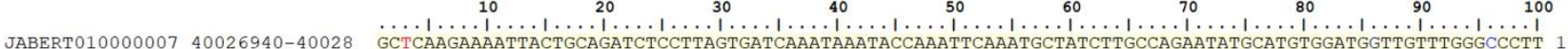
JABERT010000007_40042323-40044 GCGCAAGAAAATTACTGCAGATCTCCTTAGTGATCAAATAAATACCAAATTCAAATGCTATCTTGCCAGAATATGCATGTGGATGTTTGTT-GGCCTT 97

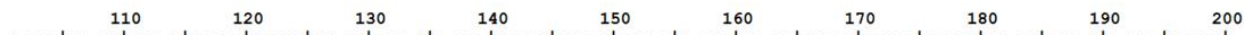
JABERT010000007_40026940-40028 TTTCAGAGAGCAATTGGTGTGGAGACCAAGGTAGTACTTGATACTGTCATTGATATATTGAGAAACTTCTTGATTTGTACAGTCTGCAATGGATATGA 200 JABERT010000007-40042323-40044 TGTCAGAGAGCAATTGGTGTGGAGACC-AAGGTAAGTACTTGATACTGTCATTGATATATTGAGAAACTTCTTGATTTGTACAGTCTGCAATGGATATGA 196

584

585

586

587

588

589

590

591

592

593

594

595

596

597

598

599

600

601

602

603

604

605

606

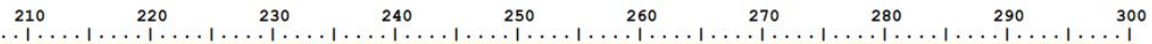
AAAGTGTCTTAGAACTAGTATTTCTATTGACTTTCATGTACTACTTTGTTTATGGGTAATCATGGGTGCTTTGAAATTATCTCCTATAGGCTACAGAGT 300

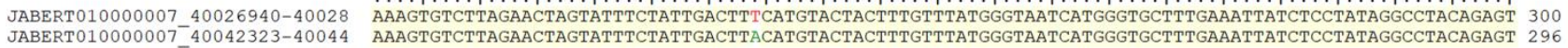

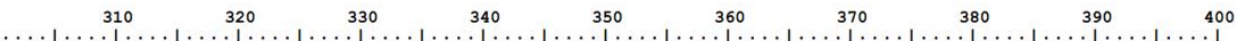
JABERT $01000000740026940-40028$ ACAAGTTTCCCTTTCCTATATTTCTATCTCCCTTTCCTTCTTCCTTCCTGCCATTTATCTTCATCTATGTATTAGTATTCTATATCTATTCTATGTCT 400 JABERT010000007-40042323-40044 ACAAGTTTTCCCTTTCCTATATTTTCTATCTCCCTTTCCTTCTTCCTTCCTGCCATTTATCTTCATCTATGTATTAGTATTCTATATCTATTCTATGTCT 396

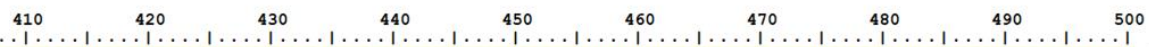

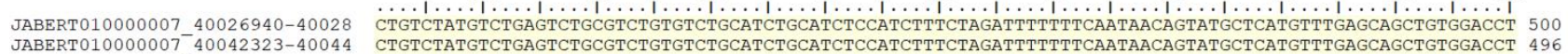

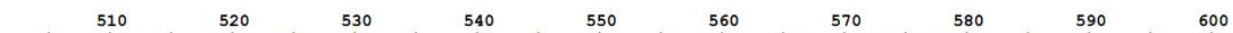

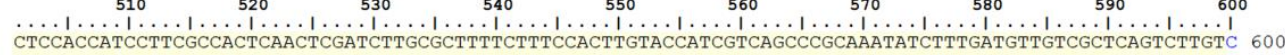

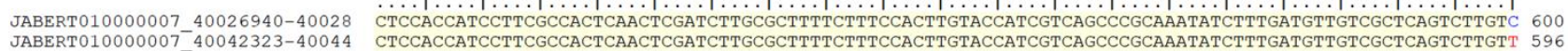

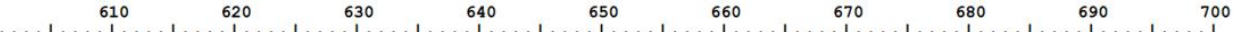
JABERT010000007_40026940-40028 TTCGGTTTGCCTCTTCCTCTGTTTCCCATCACCATCCCTGTCAGCAAGTTTTTCTCAATACTTTTACTTCTCTTCACATGACCAATAAACTTTAGTTTCC 700 JABERT010000007_40042323-40044 TTCTGTTTGCCTCTTCCTCTGTTTCCTATCACCATCCCTGTCAGCAAGTTTTTCTCAATACTTTTACTTCTCTTCACATGACCAATAAACTTTAGTTTCC 696

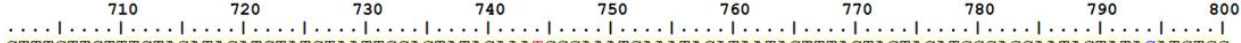

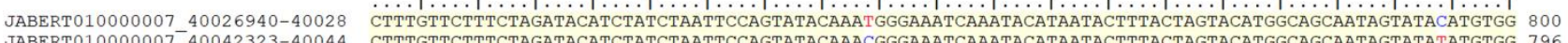
JABERT010000007_40042323-40044 CTTTGTTCTTTCTAGATACATCTATCTAATTCCAGTATACAAACGGGAAATCAAATACATAATACTTTACTAGTACATGGCAGCAATAGTATATATGTGG 796

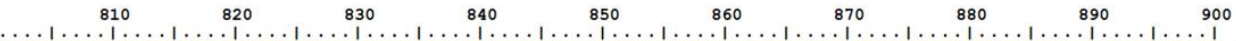
JABERT010000007_40026940-40028 AAAGGAAGAGTACTATGTAAATAAATTTTAAGTTTTTTGATTTAAGTATCAGACAATGTTAACCCCTTGATCGCGGGTGGCATCTACTATTCTGCCATG 900 JABERT010000007_40042323-40044 AAAGGAAGAGTTACTATGTAAATAAATTTTAAGTTTTTTGATTTAAGTATCAGACAATGTTAACCCCTTGATCGCGGGTGGCATCTACTATTCTGCCATG 896

$\ldots+\ldots 910 \ldots|\ldots 920 \ldots| \ldots 930 \ldots|\ldots 940 \ldots| \ldots 950 \ldots|\ldots 960 \ldots| \ldots 970 \ldots|\ldots 980 \ldots| \ldots 990 \ldots \mid \ldots 1000$ JABERT 10000007 40026940-40028 GCGATGTAGCGCTGATGCCACGGGTGGCATCCCTACAGATGTAGCCCATACTGACATACCTGTTTGGCGGACCGCACAGCCGCCAAAAA GCCCGCACAA 999 JABERT010000007_40042323-40044 GCGGTGTAGCGCTGATGCCACGGGTGGCATCCCTACAGATGTAGCCCATACTGACATACCTGTTTGGCGGACCGCACAGCCGCCAAAAAAGCCCGCACAC 996

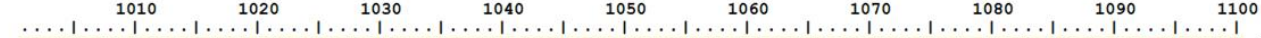
JABERT010000007_40026940-40028 AATTAGGTTAAATGGGTTAATTTTAGCAAGACAGAGCTTGGACTGGGTCCTCATTTCATGATAAATCATCTTAGTAGTCTACATATGCAATCTCTCTCAA 1099 JABERT010000007_40042323-40044 A-TTAGGTTAAATGGGT-AATTTTAGCAAGACAGAGCTTGGACTGGGTCCTCATTTCATGATAAATCATCTTAGTAGTCTACATATGCAATCGCTCTCAA 1094

JABERT010000007 40026940-40028 JABERT010000007_40042323-40044

$\begin{array}{llllllllll}1110 & 1120 & 1130 & 1140 & 1150 & 1160 & 1170 & 1180 & 1190 & 1200\end{array}$

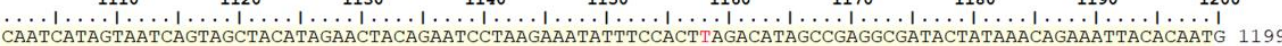
CAATCATAGTAATCAGTAGCTACATAGAACTACAGAATCCTAAGAAATATTTCCACTAAGACATAGCCGAGGCGATACTATAAACAGAAATTACACAATC 1194

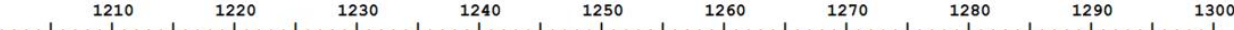

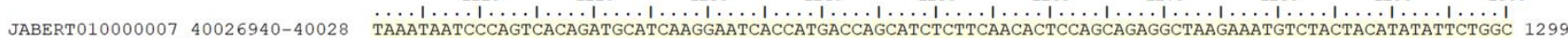
JABERT $010000007_{-}^{-40042323-40044}$ TAAATAATCCCAGTCACAGATGCATCAAGGAATCACCATGACCAGCATCTCTTCAACACTCCAGCAGAGGCTAAGAAATGTCTACTACATATATTCTGGC 1294

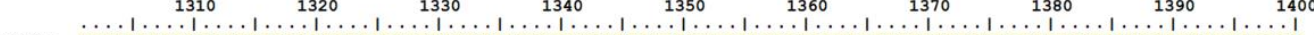
JABERT 010000007 40026940-40028 AGGAAAAAGTTAAATTTTTGATAAGTCACCAAGATGGTCTACAAATCTTCGAGGAGATCACAATGACAAAGAGCTTGTCCGGTTTTTAATTATTTTTTT 1399 JABERT010000007_-40042323-40044 AGGAAAAAGTTTAAATTTTTGATAAGTCACCAAGATGGTCTACAAATCTTCGAGGAGATCACAATGACAAAGAGCTTGTCCGGTTTTTAATTATTTTTT- 1393

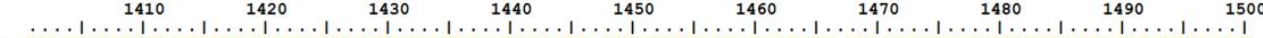
JABERT010000007_40026940-40028 CCTTTTAATTATTTTTTCAGTAACTGAAAATCACATTTTATTTTCA TATGCAACAACTGTTATGG AAAATAGATTGATGACATTAACAATGAAATACG 1497 JABERT $010000007_{-}^{-}$40042323-40044 CCTTTTAATTATTTTTTCAGTAACTGAAAATCACATTTTATTTTCAATATGCAACAACTGTTATGGGAAAATAGATTGATGACATTAACAATGAAATACG 1493

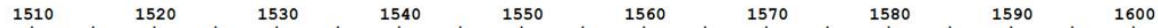

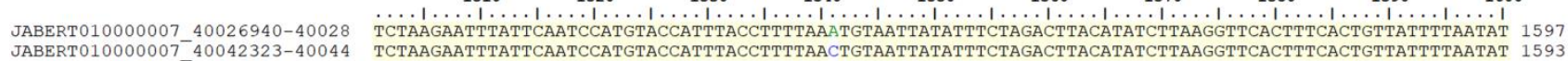
JABERT010000007_40042323-40044 $\begin{array}{llllllllll}1610 & 1620 & 1630 & 1640 & 1650 & 1660 & 1670 & 1680 & 1690 & 1700\end{array}$

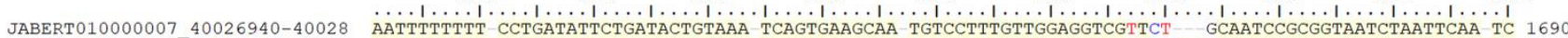
JABERT010000007_40042323-40044 AATTTTTTTTTCCTGATATTCTGATACTGTAAAATCAGTGAAGCAAATGTCCTTTGTTGGAGGTCGCTTCCTGACAATCCGCGGTAATCTAATTCAAATC 1693

607 
608 Supplementary figure S4. Alignment of the direct repetitive sequences flanking the infectious

609 IHHNV-EVE Cluster 3 located on LG7. A total 1,758 bp were aligned and share $98 \%$ identity

610 (1722/1758 bp with 21/1758 nucleotide gaps).

611 